\title{
SOPRA LA GURVATURA DI ALCUNE LINEE PRODOTTE DALL' INTERSEZIONE DI DUE SUPERFICIE DEL SECONDO GRADO
}

M E M OR I A

\section{DEL PROF. BARNABA TORTOLINI}

1. Fin dall' anno 1852 il Sig. Prof. J. Booth pubblicò nelle Transazioni filosofiche di Londra una lunga Memoria intitolata: Researches on the geometrical proporties of elliptic integrals, e nel 1854 pubblicò ancora un' altro scritto sotto to stesso titolo. Nelle citate Memorie il Sig. Booth si occupa della rettificazione e quadratura di una serie di linee a doppia curvatura provenienti dall' intersezione di due date superficie del secondo grado: il medesimo giunge a dimostrare, che in queste curve vi sono inclusc le rappresentazioni geometriche delle funzioni ellitliche delle tre note specie, ed in tutte le varietà possibili: anche io nel tom. II. di questi Annali 1859, e nel tom. IV. riprodussi alcune ricerche del Sig. Booth, ed estesi un tal argomento ad altre linee a doppia curvatura. In questa nuova Memoria mi propongo di prendere ad esame la curvatura di alcune di queste linee col determinarne i raggi delle due curvature ed il raggio di curvatura sferica, non che il luogo geometrico dei centri di curvatura sferica, quale si riduce alla linea di regresso della Superficie polare: le formole generali, delle quali faremo uso, e che per la maggior parte son dovute a Monge trovansi presentemente riportate in tutti i moderni trattati di Calcolo intinitesimale, ed io pure nel Calcolo differenziale pubblicato nel 1844 trattai con qualche nuova generalità, ed estensione la teoria delle curve a doppia curvatura; contuttociò nel corso di questa Memoria spesso mi rivolgerò a richiamare qualcuna delle formole generali riportate dal Sig. De Saint-Venant in una sua importante Memoria Sur les lignes courbes non planes, ed inserita nel fascicolo 30. del giornale della scuola politecnica per il 1845.

2: La prima linea della quale ci occuperemo a determinarne la sua curvatura sarà quella linea prodolta dall' intersezione di un paraboloide ellittico, e da un cilindro circolare aventi lo stesso asse. Se l'asse comune sia quello delle $z$ e si chiamino $k, k_{x}$ i semiparametri delle due parabole descritte nei piani $x z, y z$, sezioni principali del paraboloide, e chiamato $a$ il raggio del cilindro circolare, si avranno le due equazioni

$$
x^{2}+y^{2}=a^{2}, \quad \frac{x^{2}}{k}+\frac{y^{2}}{k_{x}}=2 z
$$

Tom. V. N. 6 . 
Come ognun vede da una tal intersezione verrà a segnarsi nella superficie del paraboloide una linea chiusa, della quale le projezioni nei piani $x z, y z$ saranno due nuove parabole: se si fosse preso un paraboloide di rivoluzione ed un cilindro ellittico di asse comune, dalla loro intersezione si ottiene pure una nuova linea chiusa, e che dal Sig. Booth fủ chiamata Ellisse logaritmica, e della quale ancora ne parleremo nel proseguimento di questa Memoria: la curva che prendiamo ora a considerare è una specie di ellisse logaritmica. Nella curvatura delle linee occorre la cognizione dei differenziali di primo ordine e di ordine superiore delle variabili $x, y, z$ e dell' arco $s$ riferendole tutte ad una variabile indipendente come funzioni: nelle precedenti equazioni adoprando la sostituzione polare

si avrà egualmente

$$
x=a \cos \theta, y=a \operatorname{sen} \theta
$$

$$
2 z=\frac{a^{2}}{k k_{1}}\left(k_{\mathrm{z}} \cos ^{2} \theta+k \operatorname{sen}^{2} \theta\right) .
$$

ove si prenderà $\theta$ per variabile indipendente. Venendo alla differenziazione successiva, si otliene

$$
\mathrm{d} x=-a \operatorname{sen} \theta \mathrm{d} \theta, \mathrm{d} y=a \cos \theta \mathrm{d} \theta, \mathrm{d} z=\frac{a^{2}}{k k_{\mathrm{x}}}\left(k-k_{\mathrm{r}}\right) \operatorname{sen} \theta \cos \theta \mathrm{d} \theta
$$

di qui per il differenziale $\mathrm{d} s$ dell' arco, e per l'equazione $\mathrm{d} s^{2}=\mathrm{d} x^{2}+\mathrm{d} y^{2}+\mathrm{d} z^{2}$, si trae

$$
\mathrm{d} s^{2}=\frac{a^{2}}{k^{2} k_{1}^{2}}\left(k^{2} k_{1}^{2}+a^{2}\left(k-k_{1}\right)^{2} \operatorname{sen}^{2} \theta \cos ^{2} \theta\right) \mathrm{d} \theta^{2}
$$

e dall' integrazione l' areo $s$ dipenderà dai trascendenti ellittici : infatti ponendo con il Sig. Booth, $2 \theta=\frac{\pi}{2}+\psi$ e ponendo di più per brevità

si ricaverà

$$
h=\frac{a^{2}}{2}\left(\frac{1}{k_{\mathrm{x}}}-\frac{1}{k}\right), \quad c^{2}=\frac{h}{\sqrt{\left(a^{2}+h^{2}\right)}}
$$

$$
s=\frac{\sqrt{ }\left(a^{2}+h^{2}\right)}{2} \int \mathrm{d} \psi \sqrt{ }\left(1-c^{2} \operatorname{sen}^{2} \psi\right)
$$

e che sarà un trascendente ellittico di seconda specie e perciò riducibile alla reltificazione dell'ellisse piana: dalla derivazione ulteriore abbiamo

$$
\begin{gathered}
\mathrm{d}^{2} x=-a \cos \theta \mathrm{d} \theta^{2}, \quad \mathrm{~d}^{2} y=-a \operatorname{sen} \theta \mathrm{d} \theta^{2} \\
\mathrm{~d}^{2} z=\frac{a^{2}}{k k_{\mathrm{x}}}\left(k-k_{\mathrm{x}}\right)\left(\cos ^{2} \theta-\operatorname{sen}^{2} \theta\right) \mathrm{d} \theta^{2}
\end{gathered}
$$


PURA ED APPLICATA.

Con questi valori componendo il differenziale di $\mathrm{ds}^{2}$, cioè

$$
\mathrm{d} s \mathrm{~d}^{2} s=\mathrm{d} x \mathrm{~d}^{2} x+\mathrm{d} y \mathrm{~d}^{2} y+\mathrm{d} z \mathrm{~d}^{2} z
$$

olterremo

$$
\mathrm{d} s \mathrm{~d}^{2} s=\frac{a^{4}\left(k-k_{\mathrm{x}}\right)^{2}}{k^{2} k_{1}^{2}}\left(\cos ^{2} \theta-\operatorname{sen}^{2} \theta\right) \operatorname{sen} \theta \cos \theta \mathrm{d} \theta^{3}
$$

La successiva derivazione porge ancora

$$
\begin{gathered}
\mathrm{d}^{3} x=a \operatorname{sen} \theta \mathrm{d} \theta^{3}, \quad \mathrm{~d}^{3} y=-a \cos \theta \mathrm{d} \theta^{3} \\
\mathrm{~d}^{3} z=\frac{-4 a^{2}\left(k-k_{\mathrm{I}}\right)}{k k_{\mathrm{I}}} \operatorname{sen} \theta \cos \theta \mathrm{d} \theta^{3}
\end{gathered}
$$

Tali sono le formole preparatorie che ei serviranno in appresso.

3. Sia $\rho$ il raggio di curvatura, e $p_{x}$ il raggio della seconda curvatura, e che suol dirsi raggio di flessione, e chiamato dal Sig. De Saint-Venant, rayon de cambrure: pongasi inoltre

$$
\begin{gathered}
\mathrm{U}=\mathrm{d} y \mathrm{~d}^{2} z-\mathrm{d} z \mathrm{~d}^{2} y, \quad \mathrm{~V}=\mathrm{d} z \mathrm{~d}^{2} x-\mathrm{d} x \mathrm{~d}^{2} z \\
\mathrm{~W}=\mathrm{d} x \mathrm{~d}^{2} y-\mathrm{d} y \mathrm{~d}^{2} x
\end{gathered}
$$

si avrà tanto per il raggio $\rho$ del circolo osculatore alla curva, quanto per il raggio $\rho_{\mathbf{x}}$ di flessione,

$$
\rho=\frac{d s^{3}}{\sqrt{\left(\mathbf{U}^{2}+V^{2}+\mathbf{W}^{2}\right)}}, \quad \rho_{\mathbf{x}}=\frac{\left(\mathbf{U}^{2}+\mathrm{V}^{2}+\mathrm{W}^{2}\right)}{\mathbf{U d}^{3} x+\mathbf{V d}^{3} y+\mathbf{W d}^{3}{ }^{2}}
$$

Nel nostro caso abbiamo per U, V, W, i valori

$$
\mathrm{U}=\frac{a^{3}}{k k_{\mathrm{x}}}\left(k-k_{\mathrm{x}}\right) \cos ^{3} \theta \mathrm{d} \theta^{3}, \quad \mathrm{~V}=-\frac{a^{3}}{k k_{\mathrm{x}}}\left(k-k_{\mathrm{x}}\right) \operatorname{sen}^{3} \theta \mathrm{d} \theta^{3}, \quad \mathrm{~W}=a^{2} \mathrm{~d} \theta^{3}
$$

d'onde

$$
\mathrm{U}^{2}+\mathrm{V}^{2}+\mathrm{W}^{2}=\frac{a^{4}}{k^{2} k_{1}^{2}}\left(k^{2} k_{1}^{2}+a^{2}\left(k-k_{1}\right)^{2}-3 a^{2}\left(k-k_{1}\right)^{2} \operatorname{sen}^{2} \theta \cos ^{2} \theta\right) \mathrm{d} \theta^{6}
$$

e perciò richiámando il valore di $d s$, si avrà per il raggio del circolo osculatore

$$
\rho=\frac{a}{k^{2} k_{1}^{2}} \frac{\left(k^{2} k_{1}^{2}+a^{2}\left(k-k_{\mathrm{I}}\right)^{2} \operatorname{sen}^{2} \theta \cos ^{2} \theta\right)^{\frac{3}{2}}}{\left(k^{2} k_{1}^{2}+a^{2}\left(k-k_{1}\right)^{2}-3 a^{2}\left(k-k_{1}\right)^{2} \operatorname{sen}^{2} \theta \cos ^{2} \theta\right)^{\frac{2}{2}}}
$$


Nei punti della curva, ove corrisponde, $\theta=0, \theta=\frac{\pi}{2}$, il raggio resta costanl., e sarà

$$
\rho^{\prime}=\frac{a k k_{\mathrm{x}}}{\sqrt{\left(k^{2} k_{1}^{2}+a^{2}\left(k-k_{\mathrm{x}}\right)^{2}\right)}}
$$

Di più dai precedenti valori di $x, y, z$, il raggio potrebbe essere espresso per una qualunque delle tre coordinate $x, y, z$; per il raggio $p_{\mathrm{x}}$ di flessione rappresentiamo. per un istante con $S$ il suo denominatore, si avrà generalmente la doppia identilà

$$
\mathrm{S}=\mathrm{Ud}^{3} x+\mathrm{Vd}^{3} y+\mathrm{Wd}^{3} z=-\mathrm{dUd}^{2} x-\mathrm{dVd}^{2} y-\mathrm{dWd}^{2} z .
$$

D'altronde dai valori di U, V, W, si trae

$$
\begin{aligned}
& \mathrm{d} \mathbf{U}=-\frac{3 a^{3}}{k k_{\mathrm{I}}}\left(k-k_{\mathrm{I}}\right) \cos ^{2} \theta \operatorname{sen} \theta \mathrm{d} \theta^{4}, \quad \mathrm{~d} \mathbf{W}=0 . \\
& \mathrm{d} \mathbf{V}=-\frac{3 a^{3}}{k k_{\mathrm{I}}}\left(k-k_{\mathrm{I}}\right) \operatorname{sen}^{2} \theta \cos \theta \mathrm{d} \theta^{4}
\end{aligned}
$$

per cui

$$
\mathrm{S}=-\frac{3 a^{4}}{k k_{\mathrm{I}}}\left(k-k_{1}\right) \operatorname{sen} \theta \cos \theta \mathrm{d} \theta^{6}
$$

d' onde per il raggio di flessione si ottiene

$$
\rho_{\mathrm{I}}=-\frac{\left(k^{2} k_{4}^{2}+a^{2}\left(k-k_{\mathrm{r}}\right)^{2}-3 a^{2}\left(k-k_{\mathrm{r}}\right)^{2} \operatorname{sen}^{2} \theta \cos ^{2} \theta\right)}{3 k k_{\mathrm{I}}\left(k-k_{\mathrm{I}}\right) \operatorname{sen} \theta \cos \theta}
$$

Qui pure il secondo membro potrebbe farsi dipendere da una qualunque delle tre coordinate $x, y, z$. A queste due grandezze $\rho, \rho_{x}$ si aggiunge dal Sig. De Saint-Venant una terza relta $\mathcal{R}$, determinata dall' equazione

$$
\frac{1}{\mathscr{R}^{2}}=\frac{1}{\rho^{2}}+\frac{1}{\rho_{1}^{2}}
$$

ed ove $\frac{1}{\Re}$ si potrebbe chiamare curvatura risultante relatiramente alle due curvature componenti $\frac{1}{\rho}, \frac{1}{\rho_{x}}$ : di più $\frac{d s}{\Re}$ rappresenterà l'angolo infinitesimo formato da due raggri di curvatura consecutivi, od anche l'angolo formato da due piani condotti per ciascun punto della curva perpendicolarmente al raggio di curvatura corrispondente: questi piani furono chiamati dal Laneret piani retiificanti, come si dirà superficie rettificante la superficie generala dal movimento dei piani rettificanti: per le stesse 
analogie si dirà pure retta rettificante in un dato punto della curva una delle generatrici di questa superficie, e coincidente con l'intersezione di due piani reltificanti consecutivi.

4. In una curva situata nello spazio dall'intersezione di due piani normali infinitamente vicini si ha la così detla retta polare, come dall' unione delle rette polari, o dal movimento del piano normale si oltiene una superficie sviluppabile, e chiamata da Monge, superficie polare: nell' intersezione di due relle polari trovasi il centro della sfera osculatrice, la quale ha un contalto di terzo ordine con la curva : il lnogo geometrico di tutti i centri provenienti ancora dall' intersezione di tre piani normali infinitamente vicini, viene ad essere la linea di regresso della superficie polare, e che dai Francesi si chiama l'arête de rebroussement.

$\dot{\mathrm{E}}$ noto che la coesistenza delle tre equazioni

$$
\begin{aligned}
& (\mathrm{X}-x) \mathrm{d} x+(\mathrm{Y}-y) \mathrm{d} y+(\mathrm{Z}-z) \mathrm{d} z=0 \\
& (\mathrm{X}-x) \mathrm{d}^{2} x+(\mathrm{Y}-y) \mathrm{d}^{2} y+(\mathrm{Z}-z) \mathrm{d}^{2} z-\mathrm{d} s^{2}=0 \\
& (\mathrm{X}-x) \mathrm{d}^{3} x+(\mathrm{Y}-y) \mathrm{d}^{3} y+(\mathrm{Z}-z) \mathrm{d}^{3} z-3 \mathrm{~d} s \mathrm{~d}^{2} s=0
\end{aligned}
$$

determina il centro di curvatura sferica, la prima delle quali appartiene al piano normale, e le altre due si ottengono dalla differenziazione successiva della prima considerando $\mathrm{X}, \mathrm{Y}, \mathrm{Z}$ come costanti, anzi tulte e tre provengono dalla successiva differenziazione dell' equazione

$$
(\mathrm{X}-x)^{2}+(\mathrm{Y}-y)^{2}+(\mathrm{Z}-z)^{2}=\mathrm{R}^{2}
$$

considerando $\mathrm{X}, \mathrm{Y}, \mathrm{Z}$, ed $\mathrm{R}$ come costanti, in questa guisa $\mathrm{R}$ sarà il raggio della sfera osculatrice ed $\mathrm{X}, \mathrm{Y}, \mathrm{Z}$ le coordinate del centro: con le denominazioni introdotte nel precedente $\mathrm{N}^{\circ} .3^{\circ}$, si avrà da quelle tre equazioni per via dell' eliminazione

$$
\begin{aligned}
& \mathrm{X}-x=\frac{3 \mathrm{Ud} s \mathrm{~d}^{2} s-\mathrm{dUd} s^{2}}{\mathrm{~S}} \\
& \mathrm{Y}-y=\frac{3 \mathrm{Vd} s \mathrm{~d}^{2} s-\mathrm{dV} \mathrm{d} s^{2}}{\mathrm{~S}} \\
& \mathrm{Z}-z=\frac{3 \mathrm{Wd} s \mathrm{~d}^{2} s-\mathrm{dW} \mathrm{d} s^{2}}{\mathrm{~S}}
\end{aligned}
$$

quali equazioni come è chiaro appartengono alla linea di regresso della superficie polare: la coesistenza poi delle sole due prime equazioni (1) ci porgerà l'equazione della della superficie polare. Relativamente poi al raggio $R$ si otterrebbero riduzioni complicate per la sostituzione dei valori di $\mathbf{X}-x, \ldots$, ed i geometri moderni 
dietro alcune considerazioni geometriche son giunti a ritrovare

$$
\mathbf{R}=\sqrt{\left(\rho^{2}+\left(\frac{\rho_{1} \mathrm{~d}_{\rho}}{\mathrm{d} s}\right)^{2}\right)}
$$

ove al solito $\rho, \rho_{\mathrm{I}}$ sono i raggi delle due curvature: questa formola si trova ancora nella citata Memoria del Sig. De Saint-Venant; contultociò nelle applicazioni riesce non di rado complicata, e che lo faremo avvertire nelle opportune occasioni.

5. Applichiamo le precedenti formole alla curva da noi considerata; così per le coordinate $\mathrm{X}, \mathrm{Y}, \mathrm{Z}$ del centro di curvatura sferica, per la sostiluzione dei valori di $\mathrm{W}, \mathrm{d} \mathbf{W}=0$, olteniamo dall' ultima dell' equazioni (2)

$$
\mathrm{Z}-\mathrm{z}=-\frac{a^{2}\left(k-k_{\mathrm{x}}\right)}{k k_{\mathrm{s}}}\left(\cos ^{2} \theta-\operatorname{sen}^{2} \theta\right)
$$

d'onde dal valore $2 z k k_{1}=a^{2}\left(k_{1} \cos ^{2} \theta+k \operatorname{sen}^{2} \theta\right)$; eliminando $\cos ^{2} \theta$, e $\operatorname{sen}^{2} \theta$, avremo

$$
\mathrm{Z}=\frac{1}{k k_{\mathrm{x}}}\left(5 k k_{\mathrm{z}} z-a^{2} k-a^{2} k_{\mathrm{\tau}}\right)
$$

od anche col sostituirvi nuovamente il valore di $z$,

$$
\mathrm{Z}=\frac{a^{2}}{2 k k_{\mathrm{r}}}\left(\left(3 k_{\mathrm{r}}-2 k\right) \cos ^{2} \theta+\left(3 k-2 k_{\mathrm{r}}\right) \operatorname{sen}^{2} \theta\right)
$$

Per le altre coordinate $X, Y$ sostituendoci $i$ valori di $U, V, d U, d W, \ldots$ si trae dopo facili riduzioni

$$
\begin{aligned}
& \mathrm{X}-x=-\frac{a \cos \theta}{k^{2} k_{\mathrm{l}}^{2}}\left(k^{2} k_{\mathrm{i}}^{2}+a^{2}\left(k-k_{\mathrm{I}}\right)^{2} \cos ^{4} \theta\right) \\
& \mathrm{Y}-y=-\frac{a \operatorname{sen} \theta}{k^{2} k_{1}^{2}}\left(k^{2} k_{\mathrm{I}}^{2}+a^{2}\left(k-k_{\mathrm{I}}\right)^{2} \operatorname{sen}^{4} \theta\right)
\end{aligned}
$$

dalle quali per la sostituzione di $x=a \cos \theta, y=a \operatorname{sen} \theta$ si avrà

$$
\mathrm{X}=-\frac{a^{3}\left(k-k_{1}\right)^{2}}{k^{2} k_{1}^{2}} \cos ^{5} \theta, \quad \mathrm{Y}=-\frac{a^{3}\left(k-k_{1}\right)^{2}}{k^{2} k_{1}^{2}} \operatorname{sen}^{5} \theta
$$

Che se si volessero sostituire nuovamente i valori di $\cos \theta$, e $\operatorname{sen} \theta$, deduciamo

$$
\mathrm{X}=-\frac{\left(k-k_{\mathrm{i}}\right)^{2}}{a^{2} k^{2} k_{1}^{2}} x^{5}, \quad \mathrm{Y}=-\frac{\left(k-k_{\mathrm{I}}\right)^{2}}{a^{2} k^{2} k_{1}^{2}} y^{5}
$$

Nei valori di $\mathrm{X}, \mathrm{Y}, \mathrm{Z}$ coesistenti si trova determinata la linea di regresso della superficie polare: la successiva eliminazione dell' angolo $\theta$ fra due delle tre espressioni 
PURA ED APPLICATA.

porgerà l'equazioni delle projezioni della curva nei tre piani ortogonali, e che verremo brevemente ad esaminare. Innanzi tutto la nuova linea trovasi al dì sotto dell' asse della $z$, almeno per valori positivi di $\operatorname{sen} \theta, \cos \theta$, come si scorge dai valori negativi di $\mathrm{X}$, ed $\mathrm{Y}$ : elevando poi al quadrato le $\mathrm{X}$, ed $\mathrm{Y}$, ed estraendo la radice quinta e sommando, olterremo l' equazione

$$
(\mathrm{X})^{\frac{2}{5}}+(\mathrm{Y})^{\frac{2}{5}}=\left(\frac{a^{6}\left(k-k_{1}\right)^{4}}{k^{4} k_{1}^{4}}\right)^{\frac{1}{5}}
$$

la quale appartiene alla projezione della nostra curva nel piano $x y$ : volendo togliere l' irrazionalità, l' equazione ascende al decimo grado, come ho fatto vedere in una mia precedente Nota. (Annali di Mat. tom. IV. N. 5.): per cui la curva è del decimo ordine: le projezioni poi nei piani $x z, y z$ saranno due curve del quinl'ordine: elevando infatti al quadrato $\mathrm{i}$ valori di $\mathrm{X}$, ed $\mathrm{Y}$ si avrà

$$
\mathrm{X}^{2}=\frac{a^{6}\left(k-k_{1}\right)^{4}}{k^{4} k_{1}^{4}} \cos ^{\mathrm{10} \theta}, \quad \mathrm{Y}^{2}=\frac{a^{6}\left(k-k_{1}\right)^{4}}{k^{4} k_{1}^{4}} \operatorname{sen}^{10} \theta
$$

Ora dal ralore di $\mathrm{Z}$ in funzione dell' angolo $\theta$, si trae

$$
\begin{aligned}
& 5\left(k-k_{1}\right) a^{2} \operatorname{sen}^{2} \theta=2 a^{2} k-3 a^{2} k_{\mathrm{z}}+2 k k_{\mathrm{z}} \mathrm{Z} \\
& 5\left(k-k_{\mathrm{I}}\right) a^{2} \cos ^{2} \theta=3 a^{2} k-2 a^{2} k_{\mathrm{z}}-2 k k_{\mathrm{1}} \mathrm{Z}
\end{aligned}
$$

Di qui per le curve di projezione nei piani $x z$ ed $y z$, abbiamo le due equazioni di quinto grado

$$
\begin{aligned}
& 5^{5}\left(k-k_{\mathrm{x}}\right) a^{4} k^{4} k_{1}^{4} \mathrm{X}^{2}=\left(3 a^{2} k-2 a^{2} k_{\mathrm{x}}-2 k k_{\mathrm{x}} \mathrm{Z}\right)^{5} \\
& 5^{5}\left(k-k_{\mathrm{1}}\right) a^{4} k^{4} k_{1}^{4} Y^{2}=\left(2 a^{2} k-3 a^{2} k_{\mathrm{1}}+2 k k_{\mathrm{x}} Z\right)^{5}
\end{aligned}
$$

Facendo infine la somma dei primi, e secondi membri, si avrà una funzione di quarto grado rapporto a, Z, l'equazione sarà divisibile per $5 a^{2}\left(k-k_{x}\right)$ ed eguali sono i coefficienti di $\mathrm{X}^{2}, \mathrm{Y}^{2}$, e rappresenterà una superficie di quart' ordine generata dalla rotazione di una curva parimenti di quart' ordine altorno l'asse della Z. Dai valori di $\mathrm{X}^{2}, \mathrm{Y}^{2}$ espressi per l'angolo $\theta$ si potrà ricavare sotto forma più compendiosa

$$
5^{4} a^{2} k^{4} k_{1}^{4}\left(\mathrm{X}^{2}+\mathrm{Y}^{2}\right)=5^{4} a^{8}\left(k-k_{1}\right)^{4}\left(1-5 \operatorname{sen}^{2} \theta \cos ^{2} \theta+5 \operatorname{sen}^{4} \theta \cos ^{4} \theta\right)
$$

ove s' intenderà eseguita la sostituzione di $\operatorname{sen}^{2} \theta, \cos ^{2} \theta$ in funzione dell' ascissa $Z$ : aggiungiamo ancora, che l' equazione della projezione del piano $x y$

$$
(\mathrm{X})^{\frac{2}{5}}+(\mathrm{Y})^{\frac{2}{5}}=\left(\frac{a^{3}\left(k-k_{1}\right)^{2}}{k^{2} k_{\imath}^{2}}\right)^{\frac{2}{5}}
$$


apparterrà pure all' equazione di un eilindro avente per asse l' asse delle Z: potremo dunque concludere che la linea di regresso della superficie polare, proverrà dall'intersezione di un cilindro di decimo ordine, e da una superficie curva di quarto, e di rivoluzione attorno l' asse delle $z$, ed aventi ambedue l' asse comune. Determinati i valori di $\mathrm{X}, \mathrm{Y}, \mathrm{Z}$, e di $\mathrm{X}-x, \mathrm{Y}-y, \mathrm{Z}-z$ sarà facile di otlenere l'espressionc del raggio di curvalura sferica senza ricorrere alla formola di sopra stabilita; infatti avremo simultaneamente

$$
\begin{aligned}
& (\mathrm{X}-x)^{2}=\frac{a^{2} \cos ^{2} \theta}{k^{4} k_{1}^{4}}\left(k^{2} k_{1}^{2}+a^{2}\left(k-k_{1}\right)^{2} \cos ^{4} \theta\right)^{2} \\
& (\mathrm{Y}-y)^{2}=\frac{a^{2} \operatorname{sen}^{2} \theta}{k^{4} k_{1}^{4}}\left(k^{2} k_{1}^{2}+a^{2}\left(k-k_{1}\right)^{2} \operatorname{sen}^{4} \theta\right)^{2} \\
& (\mathrm{Z}-z)^{2}=\frac{a^{4}}{k^{2} k_{1}^{2}}\left(k-k_{1}\right)^{2}\left(\cos ^{2} \theta-\operatorname{sen}^{2} \theta\right)^{2}
\end{aligned}
$$

d'onde posto per brevità

$$
\begin{gathered}
\mathrm{A}=\left(k^{2} k_{1}^{2}+a^{2}\left(k-k_{\mathrm{I}}\right)^{2}\right)^{2}+a^{2} k^{2} k_{1}^{2}\left(k-k_{\mathrm{I}}\right)^{2} \\
\mathbf{B}=-5 a^{2}\left(k-k_{\mathrm{I}}\right)^{2}\left(a^{2}\left(k-k_{\mathrm{I}}\right)^{2}+2 k^{2} k_{1}^{2}\right), \mathrm{C}=5 a^{4}\left(k-k_{\mathrm{x}}\right)^{4}
\end{gathered}
$$

si aurà per il raggio $\mathbf{R}$ di questa sfera

$$
\mathbf{R}=\frac{a}{k^{2} k_{\mathfrak{1}}^{2}} V\left(\mathbf{A}+\mathbf{B} \operatorname{sen}^{2} \theta \cos ^{2} \theta+\mathbf{C} \operatorname{sen}^{4} \theta \cos ^{4} \theta\right)
$$

Il ritrovato valore in forza delle relazioni di sopra stabilite per le coordinate $x, y, z$ e l' angolo $\theta$, potrebbe ridursi ad una funzione di una qualunque delle tre variabili $x, y, z$.

6. Della medesima linea di regresso della superficie polare, e che si chiama ancora la sviluppata pel piano, se ne calcola l'elemento, come prova il Sig. De SaintVenant con la formola generale

$$
\frac{\rho}{\rho_{\mathrm{I}}} \mathrm{d} s+\mathrm{d} \frac{\rho_{\mathrm{I}} \mathrm{d} \rho}{\mathrm{d} s}
$$

mà per la curva in questione è più facile il calcolarlo direttamente dalla differenziazione dei valori di $\mathrm{X}, \mathrm{Y}, \mathrm{Z}$; ottenuti al principio del precedente $\mathrm{N}$ : 5 . 
Ed avremo

$$
\begin{aligned}
\mathrm{dX} & =\frac{5 a^{3}\left(k-k_{1}\right)^{2}}{k^{2} k_{1}^{2}} \cos ^{4} \theta \operatorname{sen} \theta \mathrm{d} \theta \\
\mathrm{d} \mathrm{Y} & =-\frac{5 a^{3}\left(k-k_{\mathrm{x}}\right)^{2}}{k^{2} k_{1}^{2}} \operatorname{sen}^{4} \theta \cos \theta \mathrm{d} \theta \\
\mathrm{dZ} & =\frac{5 a^{2}\left(k-k_{\mathrm{I}}\right)}{k k_{\mathrm{x}}} \operatorname{sen} \theta \cos \theta \mathrm{d} \theta
\end{aligned}
$$

Di qui per l'elemento $\mathrm{dS}_{\mathbf{r}}$ di questa curva, si avrà

e che diviene

$$
d S_{x}=\sqrt{ }\left(d X^{2}+d Y^{2}+d Z^{2}\right)
$$

$$
\mathrm{dS}_{\mathrm{x}}=\frac{5 a^{2}\left(k-k_{\mathrm{I}}\right)}{k k_{\mathrm{x}}} \operatorname{sen} \theta \cos \theta \mathrm{d} \theta \sqrt{ }\left(k^{2} k_{1}^{2}+a^{2}\left(k-k_{\mathrm{I}}\right)^{2}\left(1-3 \operatorname{sen}^{2} \theta \cos ^{2} \theta\right)\right)
$$

Il secondo membro è integrabile parte solto forma algebrica, ed in parte per logariImi: la quarta parte della linea totale corrisponde ai limiti $\theta=0, \theta=\frac{\pi}{2}$, donde facendo $\cos 2 \theta=u$, ed insieme

$$
\mathrm{A}=4 k^{2} k_{\mathfrak{\imath}}^{2}+a^{2}\left(k-k_{\mathrm{I}}\right)^{2}, \quad \mathbf{B}=3 a^{2}\left(k-k_{\mathrm{i}}\right)^{2}
$$

si avrà

$$
\mathrm{S}_{\mathrm{I}}=\frac{3}{4} \frac{a^{2}\left(k-k_{1}\right)}{k k_{\mathrm{I}}} \int_{0}^{\mathrm{I}} \mathrm{d} u \sqrt{ }\left(\mathrm{A}+\mathrm{B} u^{2}\right)
$$

d'altronde per l' integrale indefinito abbiamo

$$
\begin{gathered}
\int \mathrm{d} u \sqrt{ }\left(\mathbf{A}+\mathbf{B} u^{2}\right)=\frac{u \sqrt{ }\left(\mathbf{A}+\mathbf{B} u^{2}\right)}{2}+\frac{\mathbf{A}}{2} \int \frac{\mathrm{d} u}{\sqrt{\left(\mathbf{A}+\mathbf{B} u^{2}\right)}} \\
\int \frac{\mathrm{d} u}{\sqrt{\left(\mathbf{A}+\mathbf{B} u^{2}\right)}}=\sqrt{\mathbf{B}} \log \left(u \sqrt{ } \mathbf{B}+\sqrt{ }\left(\mathbf{A}+\mathbf{B} u^{2}\right)\right)
\end{gathered}
$$

e perciò ai limiti $u=0, u=1$

$$
4 \mathrm{~S}_{\mathbf{I}}=\frac{5 a^{2}\left(k-k_{\mathbf{x}}\right)}{2 k k_{\mathbf{x}}}\left(\sqrt{ }(\mathbf{A}+\mathbf{B})+\sqrt{\bar{B}} \log \left(\frac{\sqrt{ } \mathbf{B}+\sqrt{ }(\mathbf{A}+\mathbf{B})}{\sqrt{\mathbf{A}}}\right)\right)
$$

Tal' è la lunghezza della sviluppata nel piano.

Tom. V. N. 6. 
Pur gli archi delle curve di projezione nei tre piani ortogonali, si avrebbero ad integrare, le tre espressioni differenziali

$$
\begin{aligned}
& \sqrt{ }\left(\mathrm{dX} \mathrm{X}^{2}+\mathrm{d} \mathrm{Y}^{2}\right)=\frac{5 a^{3}\left(k-k_{1}\right)^{2}}{k^{2} k_{1}^{2}} \operatorname{sen} \theta \cos \theta \mathrm{d} \theta \sqrt{ }\left(1-3 \operatorname{sen}^{2} \theta \cos ^{2} \theta\right) \\
& \sqrt{ }\left(\mathrm{d} \mathrm{X}^{2}+\mathrm{d} \mathrm{Z}^{2}\right)=\frac{5 a^{2}\left(k-k_{1}\right)}{k k_{\mathrm{s}}} \operatorname{sen} \theta \cos \theta \mathrm{d} \theta \sqrt{ }\left(1+\frac{a^{2}\left(k-k_{1}\right)^{2}}{k^{2} k_{1}^{2}} \cos ^{6} \theta\right) \\
& \left.\sqrt{ }\left(\mathrm{d} \mathrm{Y}^{2}+\mathrm{d} Z^{2}\right)=\frac{5 a^{2}\left(k-k_{1}\right)}{k k_{\mathrm{x}}} \operatorname{sen} \theta \cos \theta \mathrm{d} \theta \sqrt{\left(1+\frac{a^{2}\left(k-k_{1}\right)^{2}}{k^{2} k_{1}^{2}}\right.} \operatorname{sen}^{6} \theta\right)
\end{aligned}
$$

La prima s'integra per logaritmi, e le altre due s'integrano con le funzioni ellitiche: denotando con $s_{1}, s_{2}, s_{3}$ gli archi di queste tre curve, e ponendo in tulle e tre $\cos 2 \theta=u$, ayremo respetivamente

$$
\begin{gathered}
s_{1}=\frac{5 a^{3}\left(k-k_{1}\right)^{2}}{8 k^{2} k_{1}^{2}} \int \mathrm{d} u V\left(1+3 u^{2}\right) \\
s_{2}=\frac{5 a^{2}\left(k-k^{1}\right)}{4 k k_{1}} \int \mathrm{d} u V\left(1+\frac{a^{2}\left(k-k_{1}\right)^{2}}{8 k^{2} k_{1}^{2}}(1+u)^{3}\right) \\
s_{3}=\frac{5 a^{2}\left(k-k_{1}\right)}{4 k k_{1}} \int \mathrm{d} u V\left(1+\frac{a^{2}\left(k-k_{1}\right)^{2}}{8 k^{2} k_{1}^{2}}\left(1-u^{3}\right)\right.
\end{gathered}
$$

Per la prima integrando fra i limili $u=0, u=1$, avremo come sopr:

$$
4 s_{3}=\frac{5 a^{3}\left(k-k_{1}\right)^{2}}{2 k^{2} k_{1}^{2}}\left(2+\frac{1}{\sqrt{2}} \log (\sqrt{ } 3+2)\right)
$$

Nelle altre due facendo respetlivamente $1+u=v$, ed $\mathbf{1}-u=v$, l' integrazione dipenderà dai trascendenti ellillici di prima e seconda specie, e che polrebbero essere ambedue riportate all' integrazione di una formola esaminata da Legendre (Exercices de calcul tom. $\mathbf{I}^{\circ}$. pag. 55.) e che per brevità tralasciamo di sviluppare.

7. Volendo orá determinare l'equazione della Superficie polare proveniente dal movimento del piano normale avremo a prendere lo due equazioni, una appartenente al piano normale, e l'altra al piano normale successivo, vale a dire

$$
\begin{aligned}
& (\mathbf{X}-x) \mathrm{d} x+(\mathbf{Y}-y) \mathrm{d} y+(\mathbf{Z}-z) \mathrm{d} z=0 \\
& (\mathbf{X}-x) \mathrm{d}^{2} x+(\mathbf{Y}-y) \mathrm{d}^{2} y+(\mathbf{Z}-z) \mathrm{d}^{2} z-\mathrm{d} s^{2}=0
\end{aligned}
$$

e sostituire in esse $\mathrm{i}$ valori $\mathrm{di} x, y, z \mathrm{~d} x, \mathrm{~d} y, \mathrm{~d} z, \mathrm{~d} s$ in funzione dell' angolo $\theta$, $e$ 'eliminazione di questo Ira le due detle equazioni produrà un' equazione fra le 
$\mathrm{X}, \mathrm{Y}, \mathrm{Z}$ ed appartenente alla superficie polare. Richiamando i valori di $x, y, z$, e di $\mathrm{d} x, \mathrm{~d} y, \mathrm{~d} z$ di sopra riportati, e sostituiti nella prima di queste due equazioni si avrà

$k k_{\mathfrak{l}}(\mathrm{Y} \cos \theta-\mathrm{X} \operatorname{sen} \theta)+a\left(k-k_{1}\right) \mathrm{Z} \operatorname{sen} \theta \cos \theta=\frac{a^{3}\left(k-k_{\mathrm{I}}\right)}{2 k k_{\mathrm{I}}}\left(k_{\mathrm{1}} \cos ^{2} \theta+k \operatorname{sen}^{2} \theta\right) \operatorname{sen} \theta \cos \theta$

La seconda si ottiene pure dalla derivazione di questa rapporto all' angolo $\theta$; per cui il discriminante nullo rapporto ad una qualche funzione dell' angolo $\theta$ di quesl' ul= tima equazione, rappresenterà l' equazione della superficie polare: così sostituendo nuovamente i valori di $\operatorname{sen} \theta \cos \theta$ in funzione dell' ascissa $z$, si olliene un' equazione irrazionale di secondo grado rapporto a $z$, la quale, resa razionale, ascenderà all'ottavo grado rapporto alla $z$; ed il discriminante nullo di quest' equazione di ottavo grado rappresenterà l'equazione della superficie polare : noi tralasciamo una tal ricerca come di troppo complicata. Egual difficoltà presenta il determinare l'equazione della superficie sviluppabile proveniente dal movimento del piano osculatore: infalti per l'equazione di questo piano abbiamo

$(\mathrm{X}-x)\left(\mathrm{d} y \mathrm{~d}^{2} z-\mathrm{d} z \mathrm{~d}^{2} y\right)+(\mathrm{Y}-y)\left(\mathrm{d} z \mathrm{~d}^{2} x-\mathrm{d} x \mathrm{~d}^{2} z\right)+(\mathrm{Z}-z)\left(\mathrm{d} x \mathrm{~d}^{2} y-\mathrm{d} y \mathrm{~d}^{2} x\right)=0$

dalla quale per i valori dei coefficienti di sopra espressi per $\mathrm{U}, \mathrm{V}, \mathrm{W}$ ricaveremo

$$
a\left(k-k_{1}\right)\left(\mathrm{X} \cos ^{3} \theta-\mathrm{Y} \operatorname{sen}^{3} \theta\right)=a^{2}\left(k-k_{\mathrm{z}}\right)\left(\cos ^{2} \theta-\operatorname{sen}^{2} \theta\right)+k k_{\mathrm{I}}(z-\mathrm{Z})
$$

Di più dai valori di $\cos \theta, \operatorname{sen} \theta \operatorname{come}$ al $\mathrm{N}^{\circ}$. $2^{\circ}$. abbiamo

per cui

$$
\begin{aligned}
& a^{2}\left(k-k_{1}\right) \cos ^{2} \theta=k\left(a^{2}-2 k_{1} z\right) \\
& a^{2}\left(k-k_{1}\right) \operatorname{sen}^{2} \theta=k_{1}\left(2 k z-a^{2}\right)
\end{aligned}
$$

$$
a^{2}\left(k-k_{1}\right)\left(\cos ^{2} \theta-\operatorname{sen}^{2} \theta\right)=a^{2}\left(k+k_{1}\right)-4 k k_{1} z
$$

quali valori sostituiti nella precedente equazione porgono

$$
\begin{aligned}
& \sqrt{ } k^{3} \cdot \sqrt{ }\left(a^{2}-2 k_{\mathrm{x}} \mathrm{z}\right)^{3} \cdot \mathrm{X}-\sqrt{ } k_{1}^{3} \sqrt{ }\left(2 k z-a^{2}\right)^{3} \cdot \mathrm{Y} \\
= & a^{2}\left(a^{2}\left(k+k_{\mathrm{x}}\right)-k k_{\mathrm{x}} \mathrm{Z}-3 k k_{\mathrm{x}} \mathrm{z}\right) \sqrt{ }\left(k-k_{\mathrm{x}}\right) .
\end{aligned}
$$

Togliendo $\mathrm{i}$ radicali, l' equazione si eleva al sesto grado rapporto a $z$ : infatti avremo primieramente dall' elevazione al quadrato.

$$
\begin{gathered}
k^{3}\left(a^{2}-2 k_{1} z\right)^{3} \mathrm{X}^{2}+k_{1}^{3}\left(2 k z-a^{2}\right)^{3} \mathrm{Y}^{2}-2 \sqrt{ } k^{3} k_{1}^{3} \sqrt{ }\left(a^{2}-2 k_{\mathrm{I}} z\right)^{3} \sqrt{ }\left(2 k z-a^{2}\right)^{3} \mathrm{XX} \\
=a^{4}\left(k-k_{\mathrm{x}}\right)\left(a^{2}\left(k+k_{\mathrm{I}}\right)-k k_{\mathrm{1}} \mathrm{Z}-3 k k_{\mathrm{1}} z\right)^{2}
\end{gathered}
$$


dalla quale si ricaverà nuovamente l'equazione

$$
\begin{gathered}
\left(k^{3}\left(a^{2}-2 k_{\mathrm{x}} z\right)^{3} \mathrm{X}^{2}+\right. \\
\left.k_{1}^{3}\left(2 k z-a^{2}\right)^{3} \mathrm{Y}^{2}-a^{4}\left(k-k_{\mathrm{I}}\right)\left(a^{2}\left(k+k_{\mathrm{I}}\right)-k k_{\mathrm{I}} \mathrm{Z}-3 k k_{\mathrm{I}} \mathrm{z}\right)^{2}\right)^{2} \\
=4 k^{3} k_{1}^{3}\left(a^{2}-2 k_{\mathrm{z}} \mathrm{z}\right)^{3}\left(2 k z-a^{2}\right)^{3} \mathrm{X}^{2} \mathrm{Y}^{2}
\end{gathered}
$$

che ascende al sesto grado rapporto alla $z$. Il discriminante si ottiene dall' eliminazione delle due equazioni derivate rapporto alla $z$, una delle quali si riporterà all'equazione di sesto grado a radici reciproche della precedente; ma egualmente tralasciamo una tal ricerca come di troppo complicata.

8: Difficoltà somiglianti provenienti dalla teorica dell' éliminazione si presentano nella determinazione della linea luogo geometrico dei centri di curvatura. Ritenute le denominazioni di sopra stabilite è noto, che chiamando X, Y, Z le coordinate del centro di curvatura corrispondenti al punto $(x, y, z)$ della linea data, si avrả

$$
\begin{gathered}
\mathbf{X}-x=\frac{(\mathrm{Vd} z-\mathrm{Wd} y) \mathrm{d} s^{2}}{\mathrm{U}^{2}+\mathrm{V}^{2}+\mathrm{W}^{2}}, \quad \mathbf{Y}-y=\frac{(\mathrm{W} \mathrm{d} x-\mathrm{Ud} z) \mathrm{d} s^{2}}{\mathrm{U}^{2}+\mathrm{V}^{2}+\mathrm{W}^{2}} \\
\mathrm{Z}-z=\frac{(\mathrm{Ud} y-\mathrm{V} \mathrm{d} x) \mathrm{d} s^{2}}{\mathrm{U}^{2}+\mathrm{V}^{2}+\mathbf{W}^{2}}
\end{gathered}
$$

Ora sostituendo in queste equazioni $\mathrm{i}$ valori di sopra ritrovati per $\mathrm{d} x, \mathrm{~d} y, \mathrm{~d} z, \mathrm{~d} s$, $\mathbf{U}, \mathbf{V}, \mathbf{W}$, si otterranno per $\mathbf{X}$, ed $\mathbf{Y}$ due equazioni di nono grado rapporto a $\cos \theta$, $\operatorname{sen} \theta$ come per la $Z$ si otterrà un'equazione di sesto grado relativamente 0 a $\cos \theta$, o a $\operatorname{sen} \theta$ priva delle polenze impari : perciò l' equazioni nella linea luogo geometrico dei centri di curvatura dipenderanno dall' eliminazione di un' incognita, fra due equazioni una di sesto, e l'allra di nono grado, il che ci basti di aver qui indicato.

9: Dai valori generali di $X, Y, Z$ determinati dalle precedenti equazioni se ne deducono mediante la differenziazione $\mathrm{i}$ valori di $\mathrm{dX}, \mathrm{dX}, \mathrm{dZ}$ il che ci porge l'elemento dS della linea dei centri di curvalura, vale a dire

$$
\mathrm{dS}=\sqrt{ }\left(\mathrm{d} \mathbf{X}^{2}+\mathbf{d \mathbf { Y } ^ { 2 }}+\mathrm{dZ^{2 }}\right)
$$

Ora come fa vedere il Sig. De Saint-Venant nella sua Memoria più volte citata si ha generalmente

$$
\mathrm{dS}=\sqrt{\left(\frac{\rho \mathrm{d} s}{\rho_{\mathrm{I}}}\right)^{2}+\mathrm{d} \rho^{2}=\frac{\mathrm{Rd} s}{\rho_{\mathrm{I}}}}
$$

ove $\rho_{1}$, è il raggio di flessione, ed $R$ il raggio della sfera osculatrice. Ora tanto $R, p_{x}$ quanto $d s$ sono tulli espressi per le formole di sopra stabilite in funzioni 
dell' angolo $\theta$, e d $\theta$ : fatte tulte le indicate sostituzioni nel secondo membro di $\mathrm{dS}$, il valore di $\mathrm{S}$ dipenderà da un' integrale ultra ellittico.

10. Prendiamo per un' altra applicazione Ja curva che dal Sig. Booth vien chiamata Ellisse logaritmica proveniente dall' intersezione di un cilindro ellitlico, e di un paraboloide di rivoluzione aventi il medesimo asse. Sè come si è praticato nell' esempio precedente, l'asse delle $z$ sia l' asse comune alle due superficie, avremo le due equazioni simultanee

$$
\frac{x^{2}}{a^{2}}+\frac{y^{2}}{b^{2}}=1, \quad x^{2}+y^{2}=2 h z
$$

ove $a, b$ sono i due semiassi principali dell' ellisse e $2 h$ il parametro della parabola: prendendo nell' ellisse

$$
x=a \cos \theta, \quad y=b \operatorname{sen} \theta
$$

si avrà

$$
2 h z=a^{2} \cos ^{2} \theta+b^{2} \operatorname{sen}^{2} \theta
$$

e quindi per la differenziazione

$$
\begin{gathered}
\mathrm{d} x=-a \operatorname{sen} \theta \mathrm{d} \theta, \quad \mathrm{d} y=b \cos \theta \mathrm{d} \theta \\
h \mathrm{~d} z=-\left(a^{2}-b^{2}\right) \operatorname{sen} \theta \cos \theta \mathrm{d} \theta
\end{gathered}
$$

d'onde per l'elemento ds dell' ellisse logaritmica si ottiene

$$
\mathrm{d} s=\frac{\mathrm{d} \theta}{h} V\left(b^{2} h^{2}+\left(a^{2}-b^{2}\right)\left(h^{2}+a^{2}-b^{2}\right) \operatorname{sen}^{2} \theta-\left(a^{2}-b^{2}\right)^{2} \operatorname{sen}^{4} \theta\right)
$$

L'arco $s$ come già feci vedere in altra Memoria dipende da integrali riducibili ai trascendenti ellittici. Proseguendo la differenziazione nei valori di $\mathrm{d} x, \mathrm{~d} y, \mathrm{~d} z$ con ritenere $\theta$ per variabile indipendente, ricaviamo

$$
\begin{gathered}
\mathrm{d}^{2} x=-a \cos \theta \mathrm{d} \theta^{2}, \quad \mathrm{~d}^{2} y=-b \operatorname{sen} \theta \mathrm{d} \theta^{2} \\
h \mathrm{~d}^{2} z=-\left(a^{2}-b^{2}\right)\left(\cos ^{2} \theta-\operatorname{sen}^{2} \theta\right) \mathrm{d} \theta^{2}
\end{gathered}
$$

Similmente da altra differenziazione

$$
\begin{gathered}
\mathrm{d}^{3} x=a \operatorname{sen} \theta \mathrm{d} \theta^{3}, \quad \mathrm{~d}^{3} y=-b \cos \theta \mathrm{d} \theta^{3} \\
h \mathrm{~d}^{3} z=4\left(a^{2}-b^{2}\right) \operatorname{sen} \theta \cos \theta \mathrm{d} \theta^{3}
\end{gathered}
$$

Con i ritrovati valori di $\mathrm{d} x, \mathrm{~d}^{2} x \ldots$ potremo calcolare le quantità di sopra notate per U, V, W. 
11: Riprendendo infatti i valori generali di $\mathrm{U}, \mathrm{V}, \mathrm{W}$ riportati al $\mathrm{N}^{\circ}$. 3, si troverà pel nostro caso

$$
\mathbf{U}=-\frac{b\left(a^{2}-b^{2}\right)}{h} \cos ^{3} \theta \mathrm{d} \theta^{3}, \quad \mathrm{~V}=\frac{a\left(a^{2}-b^{2}\right)}{h} \operatorname{sen}^{3} \theta \mathrm{d} \theta^{3}, \quad \mathrm{~W}=a b \mathrm{~d} \theta^{3}
$$

Di qui otterremo

$$
\mathbf{U}^{2}+\mathbf{V}^{2}+\mathrm{W}^{2}=\frac{\mathrm{d} \theta^{6}}{h^{2}}\left(a^{2} b^{2} h^{2}+a^{2}\left(a^{2}-b^{2}\right)^{2} \operatorname{sen}^{6} \theta+b^{2}\left(a^{2}-b^{2}\right)^{2} \cos ^{6} \theta\right)
$$

Osservando inoltre che il valore di $\mathrm{d} s$ si potrà anche porre solto la forma

$$
\mathrm{d} s=\frac{\mathrm{d} \theta}{h} V\left(b^{2} h^{2}+h^{2}\left(a^{2}-b^{2}\right) \operatorname{sen}^{2} \theta+\left(a^{2}-b^{2}\right)^{2} \operatorname{sen}^{2} \theta \cos ^{2} \theta\right)
$$

si avrà per il raggio di curvatura $\rho$, o per il raggio del circolo osculatore alla curva, come nel citato $\mathrm{N}^{\circ} .3$.

$$
\rho=\frac{\left(b^{2} h^{2}+h^{2}\left(a^{2}-b^{2}\right) \operatorname{sen}^{2} \theta+\left(a^{2}-b^{2}\right) \operatorname{sen}^{2} \theta \cos ^{2} \theta\right)^{\frac{3}{2}}}{h^{2}\left(a^{2} b^{2} h^{2}+a^{2}\left(a^{2}-b^{2}\right)^{2} \operatorname{sen}^{6} \theta+b^{2}\left(a^{2}-b^{2}\right)^{2} \cos ^{6} \theta\right)^{\frac{4}{2}}}
$$

Facendo successivamente $\theta=0, \theta=\frac{\pi}{2}$ abbiamo i due raggi di curvatura nei due punti dell' ellisse logaritmica, corrispondenti ai vertici dell' ellisse sopra cui poggia il cilindro, e saranno

$$
\rho^{\prime}=\sqrt{\sqrt{a^{2} h^{2}+\left(a^{2}-b^{2}\right)^{2}}}, \quad \rho^{\prime \prime}=\frac{b^{3} h}{a \sqrt{b^{2} h^{2}+\left(a^{2}-b^{2}\right)^{3}}}
$$

e che si potrebbero chiamare i semiparametri dell' ellisse logaritmica. Differenziamo i valori di $\mathrm{U}, \mathrm{V}, \mathrm{W}$, sarà

$$
\begin{gathered}
\mathrm{dU}=\frac{3 b\left(a^{2}-b^{2}\right)}{h} \cos ^{2} \theta \operatorname{sen} \theta \mathrm{d} \theta^{4} \\
\mathrm{dV}=\frac{3 a\left(a^{2}-b^{2}\right)}{h} \operatorname{sen}^{2} \theta \cos \theta \mathrm{d} \theta^{4}, \quad \mathrm{dW}=0 .
\end{gathered}
$$

d'onde la quantità di sopra notata per $\mathrm{S}$, cioè

diverrà

$$
\mathrm{S}=-\mathrm{dUd}^{2} x-\mathrm{dVd}^{2} y-\mathrm{dWd}^{2} z
$$

$$
\mathrm{S}=\frac{3 a b\left(a^{2}-b^{2}\right)}{h} \operatorname{sen} \theta \cos \theta \mathrm{d} \theta^{6}
$$


Ora nel citato $\mathrm{N}^{\circ}$. 3, si ha per il raggio di flessione

$$
\rho_{\mathrm{I}}=\frac{\mathrm{U}^{2}+\mathrm{V}^{2}+\mathrm{W}^{2}}{\mathrm{~S}}
$$

e perciò ollerremo dalla sostituzione

$$
\rho_{1}=\frac{a^{2} b^{2} h^{2}+a^{2}\left(a^{2}-b^{2}\right)^{2} \operatorname{sen}^{6} \theta+b^{2}\left(a^{2}-b^{2}\right)^{2} \cos ^{6} \theta}{3 a b h\left(a^{2}-b^{2}\right) \operatorname{sen} \theta \cos \theta}
$$

Ove $\mathrm{i}$ due raggi $\rho, \rho_{\mathrm{r}}$ potrebbero anche esprimersi in funzione di una qualunque delle tre coordinate $x, y, z$. E noto she determinati $\mathrm{i}$ valori dei raggi $\rho, \rho_{\mathrm{r}}$ delle due curvalure; si polrebbe per mezzo di essi giungere al valore del raggio di curvatura sferica; ma nelle applicazioni riuscirà più comodo di farlo dipendere dalle coordinate del centro di curvatura sferica, come verremo ora a ricercare.

12. Ritenute le denominazioni di sopra stabilite ricaviamo dal valore di $\mathrm{d} s$, per la differenziazione

$$
\mathrm{d} s \mathrm{~d}^{2} s=\frac{\operatorname{sen} \theta \cos \theta \mathrm{d} \theta^{3}}{h^{2}}\left(\left(a^{2}-b^{2}\right) h^{2}+\left(a^{2}-b^{2}\right)^{2}\left(\cos ^{2} u-\operatorname{sen}^{2} u\right)\right)
$$

Di qui richiamando le formole (2) del $\mathrm{N}^{\circ} .4$, che ci porgono le coordinale del centro di curvatura sferica avremo per le ascisse $z, Z$,

$$
\begin{gathered}
\mathrm{W}=a b \mathrm{~d} \theta^{3}, \mathrm{~d} \mathrm{~W}=0, \text { e quindi } \\
\mathrm{Z}-z=\frac{1}{h}\left(\left(h^{2}+a^{2}-b^{2}\right) \cos ^{2} \theta+\left(h^{2}+b^{2}-a^{2}\right) \operatorname{sen}^{2} \theta\right)
\end{gathered}
$$

Sostituendoci i valori $\cos ^{2} \theta, \operatorname{sen}^{2} \theta$ in funzione nella $z$, si trova

$$
h(Z-z)=h^{2}-a^{2}-b^{2}+4 h z
$$

ed insieme

$$
h \mathrm{Z}=h^{2}-a^{2}-b^{2}+5 h z
$$

Sono dunque le due coordinate $z, \mathrm{Z}$ esprimibili fra di loro per una equazione lineare. Per le altre due coordinate $X, Y$ si trova dalle medesime formole (2) del $\mathrm{N}^{o}$. 4, per la sostituzione dei valori di $\mathrm{U}, \mathrm{V}, d \mathrm{~V}, \mathrm{dW}$

$$
\begin{aligned}
& \mathbf{X}-x=-\frac{\cos \theta}{a \bar{h}^{2}}\left(a^{2} h^{2}+\left(a^{2}-b^{2}\right)^{2} \cos ^{4} \theta\right) \\
& \mathbf{Y}-y=-\frac{\operatorname{sen} \theta}{b h^{2}}\left(b^{2} h^{2}+\left(a^{2}-b^{2}\right)^{2} \operatorname{sen}^{4} \theta\right)
\end{aligned}
$$


nelle quali sostituito $x=a \cos \theta, y=b \operatorname{sen} \theta$, si avrà per le coordinate $\mathrm{X}, \mathrm{Y}$

$$
\mathbf{X}=-\frac{\left(a^{2}-b^{2}\right)^{2}}{a h^{2}} \cos ^{5} \theta, \quad \mathbf{Y}=-\frac{\left(a^{2}-b^{2}\right)^{2}}{b h^{2}} \operatorname{sen}^{5} \theta
$$

Con i valori di X, Y, Z resta determinato il luogo geometrico dei centri di curvatura sferica, o ciò che torna lo stesso, la linea di regresso della superficie polare nella ellisse logarimica.

13. Per conoscere le projezioni di questa curva nei tre piani coordinali, si avranno a fare delle combinazioni binarie fra i tre valori di $\mathrm{X}, \mathrm{Y}, \mathrm{Z}$, così da $\mathrm{X}$, e da $\mathrm{Y}$ ricaviamo senza difficoltà

$$
\left(\frac{a h^{2} \mathrm{X}}{\left(a^{2}-b^{2}\right)^{2}}\right)^{\frac{2}{5}}+\left(\frac{b h^{2} \mathbf{Y}}{\left(a^{2}-b^{2}\right)^{2}}\right)^{\frac{2}{5}}=1
$$

Togliendo i radicali si giungerebbe ad un' equazione di grado decimo', per cui la curva projezione nel piano $x, y$ appartiene al decimo ordine: la stessa (1) appartiene pure ad un cilindro avente la medesima curva per direttrice, e per asse quello delle $z$. Per le altre due projezioni osserviamo primieramente che per il valore di $z$ in funzione dell' angolo $\theta$ come al $\mathrm{N}^{\circ}$. 10, si trae

$$
\left(a^{2}-b^{2}\right) \cos ^{2} \theta=2 h z-b^{2}, \quad\left(a^{2}-b^{2}\right) \operatorname{sen}^{2} \theta=a^{2}-2 h z
$$

Di qui elevando al quadrato i valori di $\mathbf{X}, \mathbf{Y}$ abbiamo egualmente

$$
\begin{aligned}
& a^{2} h^{4}\left(a^{2}-b^{2}\right) \mathrm{X}^{2}=\left(a^{2}-b^{2}\right)^{5} \cos ^{10} \theta=\left(2 h z-b^{2}\right)^{5} \\
& b^{2} h^{4}\left(a^{2}-b^{2}\right) \mathrm{Y}^{2}=\left(a^{2}-b^{2}\right)^{5} \operatorname{sen}^{10} \theta=\left(a^{2}-2 h z\right)^{5}
\end{aligned}
$$

Di più dal valore di $Z$ espresso per $z$, si trae

$$
\begin{aligned}
& 5\left(a^{2}-2 h z\right)=3 a^{2}-2 b^{2}+2 h^{2}-2 h Z \\
& 5\left(2 h z-b^{2}\right)=2 a^{2}-3 b^{2}-2 h^{2}+2 h Z
\end{aligned}
$$

d' onde olteniamo le due equazioni

$$
\begin{aligned}
& 5^{5} \cdot a^{2} h^{4}\left(a^{2}-b^{2}\right) \mathrm{X}^{2}=\left(2 a^{2}-3 b^{2}-2 h^{2}+2 h \mathrm{Z}\right)^{5} \\
& 5^{5} \cdot b^{2} h^{4}\left(a^{2}-b^{2}\right) \mathrm{Y}^{2}=\left(3 a^{2}-2 b^{2}+2 h^{2}-2 h Z\right)^{5}
\end{aligned}
$$

le quali appartengono alle projezioni della linea di regresso nei piani $x z, y z$, e che come ognuno vede sono due curve del quinto ordine: poniamo per brevità

$$
2 a^{2}-3 b^{2}-2 h^{2}=2 h \mathrm{~A}, \quad 3 a^{2}-2 b^{2}+2 h^{2}=2 h \mathrm{~B}
$$


esse divengono

$$
\begin{aligned}
& 5^{5} a^{3}\left(a^{2}-b^{2}\right) \mathrm{X}^{2}=32 h(\mathrm{~A}+\mathrm{Z})^{5} \\
& 5^{5} b^{2}\left(a^{2}-b^{2}\right) \mathrm{Y}^{2}=32 h(\mathrm{~B}-\mathrm{Z})^{5}
\end{aligned}
$$

Sommandole con sviluppare le potenze quinte otterremo la nuova equazione

$$
\begin{gathered}
5^{5} a^{2}\left(a^{2}-b^{2}\right) \mathrm{X}^{2}+5^{5} b^{2}\left(a^{2}-b^{2}\right) \mathrm{Y}^{2}-32 h\left(\mathrm{~A}^{5}+\mathrm{B}^{5}\right) \\
\left.=5.32 h\left(\left(\mathrm{~A}^{4}-\mathrm{B}^{4}\right) \mathrm{Z}+2\left(\mathrm{~A}^{3}+\mathrm{B}^{3}\right) \mathrm{Z}^{2}+2\left(\mathrm{~A}^{2}-\mathrm{B}^{2}\right) \mathrm{Z}^{3}+\mathrm{A}+\mathrm{B}\right) \mathrm{Z}^{4}\right)
\end{gathered}
$$

la quale appartiene ad una superficie di quarto ordine: possiamo dunque enunciare che Ia linea dei centri di curvalura sferica dell' ellisse logaritmica proviene dall' intersezione di un cilindro di decimo ordine, e di equazione (1); e della ritrovata superficie di quart' ordine aventi il medesimo asse. Nella riportata equazione di quarto grado, tanto le somme delle potenze, quanto la differenza di A, e B sono divisibili per $\mathbf{A}+\mathbf{B}=\frac{3\left(a^{2}-b^{2}\right)}{h}$ : d' onde tutti $\mathrm{i}$ termini dell' equazione saranno divisibili per $a^{2}-b^{2}$. Riprendendo infine $\mathrm{i}$ valori delle tre differenze

$$
\mathrm{X}-x, \mathrm{Y}-y, \mathrm{Z}-\mathrm{z}
$$

in funzion dell' angolo $\theta$, come al $\mathrm{N}^{\circ} .12$ : e facendo la somma dei quadrati si otterrebbe il raggio $\mathbf{R}$ della sfera osculatrice, e che per brevità tralasciamo di trascrivere.

14. Sarà ora facile dalle precedenti equazioni calcolare l' elemento della nostra curva, e delle sue projezioni nei tre piani coordinati, onde riconoseere da quai trascendenti dipenda la loro reltificazione. A questo oggetto riprese l'equazioni

$$
\begin{array}{ll}
\mathrm{X}=-\frac{\left(a^{2}-b^{2}\right)^{2}}{a h^{2}} \cos ^{5} \theta, & \mathrm{Y}=-\frac{\left(a^{2}-b^{2}\right)^{2}}{b h^{2}} \operatorname{sen}^{5} \theta \\
h \mathrm{Z}=h^{2}-a^{2}-b^{2}+5 h z, & 2 h z=a^{2} \cos ^{2} \theta+b^{2} \operatorname{sen}^{2} \theta
\end{array}
$$

avremo facilmente

$$
\begin{gathered}
\mathrm{dX}=-\frac{5\left(a^{2}-b^{2}\right) \cos ^{3} \theta \mathrm{d} z}{a h}, \quad \mathrm{~d} \mathrm{Y}=\frac{5\left(a^{2}-b^{2}\right) \operatorname{sen}^{3} \theta \mathrm{d} z}{b h} \\
\mathrm{~d} \mathrm{Z}=5 \mathrm{~d} z, \quad h \mathrm{~d} z=-\left(a^{z}-b^{2}\right) \operatorname{sen} \theta \cos \theta \mathrm{d} \theta
\end{gathered}
$$

dalle quali pure eliminando $\cos \theta$, $\operatorname{sen} \theta$, sarà

$$
\mathrm{d} \mathrm{X}^{2}=\frac{25 b^{2}\left(2 h z-b^{2}\right)^{3} \mathrm{~d} z^{2}}{a^{2} b^{2} h^{2}\left(a^{2}-b^{2}\right)}, \quad \mathrm{d} \mathrm{Y}^{2}=\frac{25 a^{2}\left(a^{2}-2 h z\right)^{3} \mathrm{~d} z^{2}}{a^{2} b^{2} h^{2}\left(a^{2}-b^{2}\right)}, \quad \mathrm{dZ} \mathrm{Z}^{2}=25 \mathrm{~d} z^{2}
$$

Tom. v. N. 6 . 
Formando con queste tre espressioni, l' elemento $d_{S_{\mathbf{I}}}$ della curva vale a dire

$$
d S_{1}=\sqrt{ }\left(d X^{2}+d Y^{2}+d Z^{2}\right)
$$

si vede che il coefficiente di $\mathrm{d} z$ è un radicale di secondo ordine contenente un quadrinomio di terzo grado rispetto alla $z$, sarà dunque l' integrale $S_{I}$ riducibile ai trascendenti ellittici: nella stessa guisa per le tre curve di projezione, gli elementi $\mathrm{d} s_{3}, \mathrm{~d} s_{2}, \mathrm{ds}_{3}$ saranno

$$
\mathrm{d} s_{\mathrm{t}}=\sqrt{ }\left(\mathrm{d} \mathrm{X}^{2}+\mathrm{d} \mathrm{Y}^{2}\right), \quad \mathrm{d} s_{\mathrm{s}}=\sqrt{ }\left(\mathrm{dX}^{2}+\mathrm{d} \mathrm{Z}^{2}\right), \quad \mathrm{d} s_{3}=\sqrt{ }\left(\mathrm{d} \mathrm{Y}^{2}+\mathrm{dZ} \mathrm{Z}^{2}\right)
$$

e sostituili $\mathrm{i}$ valori in funzione $\mathrm{di} z, \mathrm{e} d z, \mathrm{i}$ coefficienti $\mathrm{di} \mathrm{d} z$ sono tre radieali di quadrinomi di terzo grado rispetto alle $z$, per cui la retlificazione di queste curve dipenderà egualmente dai trascendenti ellittici. Nel primo esempio da noi scelto, e relativo all' intersezione di un cilindro circolare con un paraboloide ellitlico furono indicate nei $\mathrm{N}^{i} .7^{\circ}$, ed $8^{\circ}$ quali fossero l' equazioni generali, dalle quali dipendano, la superficie polare, la superficie proveniente dal movimento del piano osculatore, e la linea luogo geometrico dei centri di curvatura: e fù veduto che i problemi sí ríducevano all' eliminazione di una incognita fra due equazioni di grado elevato, il che rende assai complicate l' equazioni risultanti di quelle date superficie e linee. Queste difficoltà quasi nello stesso grado si riproducono, volendo fare indagini somiglianti per l' ellisse logaritmica, per cui basti di aver qui indicato questa ulteriore applicazione.

15. L' analogia che passa fra l' ellisse, e l' iperbola, ci porge subito l'idea della curva proveniente dall' intersezione di un cilindre iperbolico, e di una paraboloide di rivoluzione con lo stesso asse: questa nuova curva si potrà chiamare Iperbola logaritmica conforme alle denominazioni usate dal Sig. Booth: come è chiaro l'equazioni simultanee sono

$$
\frac{x^{2}}{a^{2}}-\frac{y^{2}}{b^{2}}=1, \quad x^{2}+y^{2}=2 h z
$$

Per l'iperbola come è noto si prende

$$
x=a \sec \theta, \quad y=b \operatorname{tang} \theta
$$

d' onde per la terza coordinata

$$
2 h z \cos ^{2} \theta=a^{2}+b^{2} \operatorname{sen}^{2} \theta
$$

Senza riportare tutte le operazioni del calcolo, e se è permesso di estendere l' ana logia di queste due curve come si fà nell' ellisse, e nell' iperbola per la mulazione 
di $-b^{2}$ in luogo di $b^{2}$, potremo dire che per la linea di regresso dell' iperbola logaritmica avremo le tre equazioni

$$
\begin{gathered}
\mathrm{X}=-\frac{\left(a^{2}+b^{2}\right)^{2}}{a h^{2}} \sec ^{5} \theta, \quad \mathrm{Y}=-\frac{\left(a^{2}+b^{2}\right)^{2}}{b h^{2}} \operatorname{tang}^{5} \theta \\
h \mathrm{Z}=h^{3}-a^{2}+b^{2}+5 h z
\end{gathered}
$$

Di qui per le tre curve di projezione nei tre piani coordinati s' incontreranno curve analoghe, e dello stesso ordine a quelle ritrovate per l'ellisse logaritmica. La rettificazione di tutle le indicate curve dipenderà pure dai trascendenti ellittici. Infine tulti i risultati ottenuti per l' ellisse logaritmica, potranno con la convenevole modificazione adaltarsi all' Iperbola logaritmica.

16. Facendo intersecare un cilindro ellittico con una sfera concentrica, si otliene una curva conosciuta solto il nome di Ellisse sferica, della quale le sue projezioni nei tre piani coordinati sono incluse nelle due equazioni

$$
\frac{x^{2}}{a^{2}}+\frac{y^{2}}{b^{2}}=1, \quad x^{2}+y^{2}+z^{2}=r^{2}
$$

L' ellisse sferica, $0^{\circ}$ più generalmente una conica sferica proviene ancora dall' intersezione di un cono di secondo grado con una sfera concentrica; e le proprietà di questa curva unitamente ad altre curve sferiche furono già da me sviluppate in diverse precedenti Memorie, nelle quali mi proposi di rintracciarne la rellificazione, e la curvatura delle dette linee: ci basterà rammemorare, che la rettificazione dell'ellisse sferica dipende da un trascendente ellittico di terza specie: determinai $i$ raggi delle due curvature, ed era facile il concludere che per tutte le curve sferiche il raggio della sfera osculatrice coincide con il raggio della sfera ove si trovano descritte. (Vedasi una mia Memoria pubblicata nel 1849 nelle Memorie della Società Italiana, e ristampata nel tom. $1^{\circ}$ Annali di Scienze Matematiche e Fisiche 4850: vedasi pure un' altra mia Memoria pubblicata nel giornale Areadico nel 1845, ed una pubblicata negli Alti dell' Accademia de' nuovi Lincei pel 1852).

17. Considerando altre curve indicate dal Sig. Booth, si prenda un paraboloide ellittico, ed una sfera avente il medesimo asse con l'origine al vertice del paraboloide, avremo le due equazioni

$$
\frac{x^{2}}{k}+\frac{y}{k_{x}}=2 z, \quad x^{2}+y^{2}+z^{2}=2 r z
$$

La curva proveniente dall' intersezione di queste due superficie, essendo una curva 
sferica, si avrà per le coordinate della linea di regresso, e per il raggio della sfera osculatrice

$$
\mathrm{X}=0, \quad \mathrm{Y}=0, \quad \mathrm{Z}=\boldsymbol{r}=\mathrm{R}
$$

come deve essere nelle curve sferiche: riassumendo l' equazioni delle due superficie, $\dot{\mathrm{e}}$ evidente che $\mathrm{i}$ valori delle $x, y, z$ atti a verificarle saranno

$$
\begin{gathered}
x=\sqrt{ } 2 k z \cdot \cos \theta, \quad y=\sqrt{ } 2 k_{x} z \cdot \operatorname{sen} \theta \\
z=2\left(r-k+k \operatorname{sen}^{2} \theta-k_{x} \operatorname{sen}^{2} \theta\right)
\end{gathered}
$$

dalle quali si trae egualmente

$$
2\left(k-k_{\mathrm{z}}\right) \operatorname{sen}^{2} \theta=z-2 r+2 k, \quad 2\left(k-k_{\mathrm{s}}\right) \cos ^{2} \theta=2 r-2 k_{\mathrm{z}}-z
$$

La sostituzione di $\operatorname{sen} \theta, \cos \theta$ nei valori di $x, y$, li renderà dipendenti dalla sola $z$ : Cosi dalla differenziazione si ha

$$
\begin{aligned}
& \mathrm{d} x=\frac{\sqrt{ } k}{\sqrt{\left(k-k_{\mathrm{x}}\right)}} \sqrt{\frac{\left(r-k_{\mathrm{x}}-z\right) \mathrm{d} z}{\left(2 r z-2 k_{\mathrm{x}} z-z^{2}\right)}}, \quad \mathrm{d} y=\frac{\sqrt{ } k_{\mathrm{x}}}{\sqrt{\left(k-k_{\mathrm{x}}\right)}} \frac{(z-r+k) \mathrm{d} z}{\sqrt{\left(z^{2}-2 r z+2 k z\right)}} \\
& \mathrm{d} z=4\left(k-k_{\mathrm{z}}\right) \operatorname{sen} \theta \cos \theta \mathrm{d} \theta
\end{aligned}
$$

Formando ora per il valore dell' elemento ds dell' arco, l' equazione

$$
\mathrm{d} s^{2}=\mathrm{d} x^{2}+\mathrm{d} y^{2}+\mathrm{d} z^{2}
$$

porge a riduzioni eseguite

$$
\mathrm{d} s=2 \mathrm{~d} \theta \cdot \frac{\sqrt{ }\left(k_{\mathrm{x}}(r-k)^{2} \cos ^{2} \theta+k\left(r-k_{\mathrm{z}}\right)^{2} \operatorname{sen}^{2} \theta\right)}{\sqrt{\left((r-k) \cos ^{2} \theta_{1}^{\prime}+\left(r-k_{\mathrm{x}}\right) \operatorname{sen}^{2} \theta\right)}}
$$

L' integrale dipende dai trascendenti ellittici, per il quale ci dispensiamo di ruportarne le riduzioni date dalla trasformazione della variabile.

18. La successiva differenziazione dei valori $\mathrm{di} \mathrm{d} x, \mathrm{~d} y, \mathrm{~d} z$, ci porgerà i valori delle quantità che nel parag. $3^{\circ}$ abbiamo notato con $U, V, W$ e che occorrono nelle espressioni dei raggi delle due curvature, e nelle equazioni dalle quali dipende il luogo geometrico dei centri di curvatura: così se per brevità si ponga

$$
\mathrm{M}=2 r z-2 k_{\mathrm{z}} z-z^{2}, \quad \mathrm{~N}=z^{2}-2 r z+2 k z
$$


si troverà

$$
\begin{aligned}
& \mathrm{d}^{2} x=\frac{\sqrt{ } k}{\sqrt{\left(k-k_{\mathrm{s}}\right)}}\left(\frac{\left(r-k_{\mathrm{x}}-z\right) \mathrm{d}^{2} z}{\sqrt{\mathrm{M}}}-\frac{\left(r-k_{\mathrm{x}}\right)^{2}}{\sqrt{\mathrm{M}^{3}}} z^{2}\right) \\
& \mathrm{d}^{2} y=\sqrt{\sqrt{\left(k-k_{\mathrm{x}}\right)}}\left(\frac{(z-r+k) \mathrm{d}^{2} \mathrm{z}}{\sqrt{\mathrm{N}}}-\frac{(r-k)^{2} \mathrm{~d} z^{2}}{\sqrt{\mathrm{N}^{3}}}\right)
\end{aligned}
$$

Di qui per i valori di $U, V, W$, otterremo primieramente

$$
\begin{aligned}
& \mathrm{U}=\sqrt{ } \frac{\sqrt{ } k_{\mathrm{x}}}{\left(k-k_{\mathrm{x}}\right)}\left(\frac{(r-k)^{2} \mathrm{~d} z^{3}}{\sqrt{\mathrm{N}^{3}}}\right), \quad \mathrm{V}=-\sqrt{\sqrt{\left(k-k_{\mathrm{x}}\right)}}\left(\frac{\left(r-k_{\mathrm{x}}\right)^{2} \mathrm{~d} z^{3}}{\sqrt{\mathrm{M}^{3}}}\right) \\
& \mathrm{W}=\frac{\sqrt{ } k k_{\mathrm{x}}}{k-k_{\mathrm{x}}}\left(\frac{\left(r-k_{\mathrm{x}}\right)^{2}(z-r+k)}{\sqrt{\mathrm{N}} \cdot \sqrt{\mathrm{M}^{3}}}-\frac{(r-k)^{2}\left(r-k_{\mathrm{x}}-z\right)}{\sqrt{\mathrm{M}} \cdot \sqrt{\mathrm{N}^{3}}}\right) \mathrm{d} z^{3}
\end{aligned}
$$

Se successivamente si sostituiscano i valori $\mathrm{di} z, \mathrm{~d} z$ in funzione dell' angolo $\theta$, e nuovamente ancora si riproducano $i$ valori $\mathrm{di}^{2} \operatorname{sen}^{2} \theta, \cos ^{2} \theta$ espressi per $z$, otterremo a tulte riduzioni eseguite

$$
\begin{gathered}
\mathrm{U}=\frac{64\left(k-k_{\mathrm{z}}\right)(r-k)^{2} \sqrt{ } k_{\mathrm{x}} \cos ^{3} \theta \mathrm{d} \theta^{3}}{\sqrt{8 z^{3}}} \\
\mathrm{~V}=\frac{-64\left(k-k_{\mathrm{x}}\right)\left(r-k_{\mathrm{x}}\right)^{2} \sqrt{ } k \operatorname{sen}^{3} \theta \mathrm{d} \theta^{3}}{\sqrt{8 z^{3}}} \\
\mathrm{v}=\frac{64 \sqrt{ } k k_{\mathrm{x}}}{8 \mathrm{z}}\left(\left(2 r-k-k_{\mathrm{z}}\right) \mathrm{z}-3(r-k)\left(r-k_{\mathrm{x}}\right)\right) \mathrm{d} \theta^{3}
\end{gathered}
$$

Facendo la somma dei quadrati e sostituendo i valori delle potenze pari di $\operatorname{sen} \theta$, $\cos \theta$ in funzione della $z$, si troverà

ove si è posto

$$
\sqrt{ }\left(\mathrm{U}^{2}+\mathrm{V}^{2}+\mathrm{W}^{2}\right)=\frac{8 \mathrm{~d} \theta^{3}}{\sqrt{z^{3}}} \sqrt{ }\left(\mathrm{A} z^{3}+\mathrm{B} z^{2}+\mathrm{C} z+\mathrm{D}\right)
$$

$$
\begin{gathered}
\mathbf{A}=\left(r-k k_{\mathrm{z}}\right)^{2}, \quad \mathrm{~B}=-\delta r(r-k)\left(r-k_{\mathrm{z}}\right)\left(r^{2}-k k_{\mathrm{z}}\right) \\
\mathrm{C}=3(r-k)^{2}\left(r-k_{\mathrm{x}}\right)^{2}\left(4 r^{2}-k k_{\mathrm{y}}\right), \quad \mathrm{D}=-8 r(r-k)^{3}\left(r-k_{\mathrm{z}}\right)^{3}
\end{gathered}
$$

Resta a calcolarsi la quantità denotata per $\mathrm{S}$, vale a dire

$$
\mathrm{S}=-\mathrm{dUd}^{2} x-\mathrm{dVd}^{2} y-\mathrm{dWd}^{2} z
$$

Eseguite le differenziazioni di $\mathrm{U}, \mathrm{V}, \mathrm{W}$, si avrà per la sostituzione, e riduzione

$$
\mathrm{S} . z^{3}=-6.64\left(k-k_{\mathrm{x}}\right)(r-k)^{2}\left(r-k_{\mathrm{x}}\right)^{2} \sqrt{ } k k_{\mathrm{z}} \operatorname{sen} \theta \cos \theta \mathrm{d} \theta^{6}
$$


Tutti i riportati valori di $\mathrm{U}, \mathrm{V}, \mathrm{W}$, e di $\mathrm{S}$ son quelli che ci daranno i raggi $\rho, \rho_{\mathrm{r}}$ delle due curvature: di più sostituili nell' equazioni riportate al $\mathrm{N}^{\circ} .8^{\circ}$. si olterrebbero le coordinate $\mathrm{X}, \mathrm{Y}, \mathrm{Z}$, dei centri di curvatura espresse in funzione della coordinata $z$ della linea data: Ma i risultati provenienti da tutte queste sostituzioni non sono suscettivi di riduzioni semplici il che ci basti di aver qui indicato.

19. Scegliendo per ultima applicazione la curva prodotta da un cono ellittico, e da un paraboloide circolare al comun verlice, o centro: potremo prendere con il Sig. Booth, le due equazioni della forma

$$
a^{2} x^{2}+b^{2} y^{2}=4 h^{2} z^{2}, \quad x^{2}+y^{2}=2 h z
$$

Otteniamo cosi per la projezione nel piano $x y$ una curva di quart' ordine

$$
\left(x^{2}+y^{2}\right)^{2}=a^{2} x^{2}+b^{2} y^{2}
$$

la quale come è noto rappresenta la projezione ortogonale del centro dell' ellisse di semiassi $a, b$ sulle sue tangenti. Eliminando di più successivamente la $y$, e la $x$, avremo le due curve di secondo grado per projezioni nei piani $x z$, ed $y z$ di equazioni

$$
\left(a^{2}-b^{2}\right) x^{2}=2 h\left(2 h z^{2}-b^{2} z\right), \quad\left(a^{2}-b^{2}\right) y^{2}=2 h\left(a^{2} z-2 h z^{2}\right)
$$

nelle quali per $a>b$ la prima apparterrà ad un' iperbola, e la seconda ad un' ellisse. Prendendo la $z$ per variabile indipendente si ha dalla differenziazione

$$
\left(a^{2}-b^{2}\right) x \mathrm{~d} x=h\left(4 h z-b^{2}\right) \mathrm{d} z, \quad\left(a^{2}-b^{2}\right) y \mathrm{~d} y=h\left(a^{2}-4 h z\right) \mathrm{d} z .
$$

Formando con $\mathrm{d} x, \mathrm{~d} y, \mathrm{~d} z$, l' espressione

$$
\mathrm{d} s=\sqrt{ }\left(\mathrm{d} x^{2}+\mathrm{d} y^{2}+\mathrm{dz} z^{2}\right)
$$

e ridotla la quantità solto il vincolo radicale ad una funzione di $z$, l' arco $s$ dipenderà dagli integrali ultra ellittici : gli archi poi della curva projezione nel piano $x z$ dipenderanno dagli integrali ellittci di prima, e terza specie come già da lungo tempo lo dimostrai per questa curva, e per altre somiglianti in una mia Memoria pubblicata nel giornale Arcadico nel 1844.

20 . Supponendo sempre la $z$ variabile indipendente avremo dalla differenziazione successiva

$$
\left(a^{2}-b^{2}\right)^{2} x^{3} \mathrm{~d}^{2} x=-h^{2} b^{4} \mathrm{~d} z^{2}, \quad\left(a^{2}-b^{2}\right)^{2} y^{3} \mathrm{~d}^{2} y=-h^{2} a^{4} \mathrm{~d} z^{2}
$$

Di qui le quantità denotate di sopra per U, V, W divengono

$$
\mathrm{U}=-\mathrm{d} z \mathrm{~d}^{2} y, \quad \mathrm{~V}=\mathrm{d} z \mathrm{~d}^{2} x ; \quad \mathrm{W}=\mathrm{d} x \mathrm{~d}^{2} y-\mathrm{d} y \mathrm{~d}^{2} x
$$


e si avià dalla sostituzione

$$
\begin{gathered}
\mathrm{U}=\frac{h^{2} a^{4} \mathrm{~d} z^{3}}{\left(a^{2}-b^{2}\right)^{2} y^{3}}, \quad \mathrm{~V}=-\frac{h^{2} b^{4} \mathrm{~d} z^{3}}{\left(a^{2}-b^{2}\right)^{2} x^{3}} \\
\mathrm{~W}=\frac{4 h^{5}\left(3 a^{2} b^{2}-4 h\left(a^{2}+b^{2}\right) z\right) \mathrm{d} z^{3}}{\left(a^{2}-b^{2}\right)^{3} x^{3} y^{3}}
\end{gathered}
$$

Differenziando ancora i ritrovati valori delle $\mathrm{U}, \mathrm{V}, \mathrm{W}$, si ricaverà

$$
\begin{gathered}
\mathrm{d} \mathrm{U}=-\frac{3 h^{3} a^{4}\left(a^{2}-4 h z\right) \mathrm{d} z^{4}}{\left(a^{2}-b^{2}\right)^{3} y^{5}} \\
\mathrm{dV}=\frac{3 h^{3} b^{4}\left(4 h z-b^{2}\right) \mathrm{d} z^{4}}{\left(a^{2}-b^{2}\right)^{3} x^{5}} \\
\mathrm{dW}=\frac{48 h^{5} z^{3}\left(4 h z-b^{2}\right)\left(a^{2}-4 h z\right)\left(h\left(a^{2}+b^{2}\right) z-a^{2} b^{2}\right) \mathrm{d} z^{4}}{\left(a^{2}-b^{2}\right)^{5} x^{5} y^{5}}
\end{gathered}
$$

A questi valori possiamo aggiungere, la quantità di sopra denotata per $\mathrm{S}$, vale a dire

$$
\mathrm{S}=-\mathrm{dU} \mathrm{d}^{2} x-\mathrm{dV} \mathrm{d}^{2} y-\mathrm{dW} \mathrm{d}^{2} z
$$

e che nel nostro caso diverrà

$$
\mathrm{S}=\frac{12 h^{7} a^{4} b^{4} z^{2} \cdot \mathrm{d} z^{6}}{\left(a^{2}-b^{2}\right)^{5}} \frac{x^{5} y^{5}}{}
$$

Tali sono le formole preparatorie con le quali si può giungere ai valori dei raggi delle due curvature, ed al raggio di curvatura sferica : le medesime formole ci farebbero di più conoscere $l^{\prime}$ equazioni rappresentanti il luogo geometrico tanto dei centri di curvatura, quanto dei centri di curvatura sferica; aggiungiamo infine che le medesime formole ci porgerebbero l' equazioni dalle quali dipende la superficie polare, e la superficie proveniente dal movimento del piano oseulatore. Ma tutte le ricerche qui indicate conducono, come nella maggior parte dei casi precedenti a risultati assai complicati, per curi cessiamo di farne uno sviluppo maggiore.

Roma 20 Decembre 1863. 